\title{
Soliton pinning by long-range order in aperiodic systems
}

\author{
Francisco Domínguez-Adame \\ Departamento de Física de Materiales, Facultad de Físicas, Universidad Complutense, E-28040 Madrid, Spain \\ Angel Sánchez \\ Departamento de Matemáticas, Escuela Politécnica Superior, Universidad Carlos III, C./ Butarque 15, \\ E-28911 Leganés, Madrid, Spain \\ Yuri S. Kivshar \\ Optical Sciences Centre, Australian National University, Australian Capital Territory 0200 Canberra, Australia
}

(Received 22 March 1995; revised manuscript received 30 May 1995)

\begin{abstract}
We investigate propagation of a kink soliton along inhomogeneous chains with two different constituents, arranged either periodically, aperiodically, or randomly. For the discrete sine-Gordon equation and the Fibonacci and Thue-Morse chains taken as examples, we have found that the phenomenology of aperiodic systems is very peculiar: On the one hand, they exhibit soliton pinning as in the random chain, although the depinning forces are clearly smaller. In addition, solitons are seen to propagate differently in the aperiodic chains than on periodic chains with large unit cells, given by approximations to the full aperiodic sequence. We show that most of these phenomena can be understood by means of simple collective coordinate arguments, with the exception of long-range order effects. In the conclusion we comment on the interesting implications that our work could bring about in the field of solitons in molecular (e.g., DNA) chains.

PACS number(s): $03.20 .+\mathrm{i}, 85.25 . \mathrm{Cp}, 87.15 .-\mathrm{v}, 61.44 .+\mathrm{p}$
\end{abstract}

The subtle interplay between nonlinearity and disorder is being laboriously unveiled throughout the past few years [1]. A rich diversity of phenomena stems from such interaction, their manifestations being found in a number of systems ranging from condensed matter physics to biophysics [2]. A number of models have been set forth which capture the essential ingredients of those systems while enjoying a canonical, nonspecific view of the problem. Among the most successful of these models, the sine-Gordon (SG) equation is particularly remarkable both for its range of applicability and the possibilities it opens for study either in the continuous or discrete version. Some of the physical situations well modeled by this equation are, for instance, Josephson junctions [3], Josephson junction arrays (JJA's) [4,5], or DNA promoter dynamics $[6,7]$. Recent experiments have pointed out the relevance of this kind of model in DNA-related molecules $[6,8]$ as well as in proteins [9]. Indeed, solitons are a quite general phenomenon appearing in organic molecules [10]. Importantly, many realistic systems like DNA chains are neither periodic nor random, being inherently close to quasiperiodic or aperiodic systems, so that the effects of long-range order may change the dynamics of nonlinear excitations. DNA sequences have been shown to exhibit longrange correlations in the past few years [11], and hence the interest of a study of the type we report here.

In this Rapid Communication we concern ourselves with the problem of the behavior of kink solitons on lattices consisting of two different components, thereby focusing on issues inherently discrete similar to those of DNA or JJA dynamics. Our main aim here is to learn about the phenomenology of soliton propagation as a function of the order of the underlying lattice. We consider three main possibilities for our binary chain: periodic, aperiodic, and random, which represent, respectively, full order, long-range order, and pure disorder. We show in the following that, while the periodic lattice exhibits basically the same features as the homogeneous case, the two nonperiodic systems present characteristics of their own. We further discuss how most of our results can be understood within the framework of the collective coordinate technique [12] (see also the review [13] and references therein). Notwithstanding that analytical insight, we have also found effects that cannot be interpreted in terms of such a particlelike behavior, and we have been able to associate those to the long-range order characteristics of aperiodic chains.

The model we use as our working example is a damped, dc driven, discrete SG equation given by

$$
\ddot{u}-\frac{1}{a^{2}}\left(u_{n+1}-2 u_{n}+u_{n-1}\right)+V_{n} \sin u_{n}+\alpha \dot{u}=F,
$$

where overdot means time derivative, $a$ is the lattice spacing, and $n$ runs over the lattice sites $n=1, \ldots, N$. The coefficient in front of the on-site potential, $V_{n}$, is directly related to the physical properties of the application one is interested in: Thus, it has to do with local critical currents in Josephson devices, or with the strength of hydrogen bonds between complementary bases in DNA models. In the following, we will allow $V_{n}$ to take on only two values, $V_{a}$ and $V_{b}$. Moreover, by an appropriate rescaling, it is possible to fix $V_{a}=1$, and so this will be done hereafter. The spatial arrangement of the two kinds of values will be chosen to be either periodic, aperiodic, or random. As our aperiodic models, we pick two standard choices, namely, the Fibonacci and the Thue-Morse chains. They are generated starting from two basic units $A$ and $B$ using the following inflation rules: $A \rightarrow A B, \quad B \rightarrow A$ for the Fibonacci chain and $A \rightarrow A B$, $B \rightarrow B A$ for the Thue-Morse chain. In this way, finite and self-similar aperiodic chains are obtained by $n$ successive applications of these rules, with $N=F_{n}$ sites for the Fi- 
bonacci lattice and $N=2^{n}$ sites for the Thue-Morse lattice. Here $F_{n}=F_{n-1}+F_{n-2}$ with $F_{0}=F_{1}=1$ are the Fibonacci numbers. The number of $A$ sites in the lattice is $\sim \tau N$ in the Fibonacci case and $N / 2$ in the Thue-Morse case, where $\tau=\lim _{n \rightarrow \infty}\left(F_{n-1} / F_{n}\right)=(\sqrt{5}-1) / 2$ is the inverse golden mean. Both chains have been used in very many contexts to model aperiodic ordering which, in spite of previous, more naive ideas, it is not something intermediate between periodic and random systems (see, e.g. [14], and references therein).

To characterize the dynamics of kink solitons on these systems, we have numerically simulated Eq. (1) by means of a fifth-order adaptive-stepsize Runge-Kutta routine [15] which has been shown to be an accurate procedure (see [16] and references therein). In the homogeneous case $\left(V_{n}=V_{a}=1\right)$, it has long been known [12] that if a soliton initially at rest evolves according to Eq. (1) with $F>0$, it eventually reaches a steady state in which it propagates along the chain with velocity

$$
v=\left[1+\left(\frac{4 \alpha}{\pi F}\right)^{2}\right]^{-1 / 2},
$$

with the opposite sign if $F<0$. This result was found by means of a standard collective coordinate calculation, and the derivation can be found in [12]. As another check of our simulations, we compared their outcome to this prediction and found an agreement better than $1 \%$ for all studied cases. We will also make use of this expression, although in a modified form: If one assumes $V_{n}$ is constant and given by the average of $V_{a}$ and $V_{b}$ weighted by their concentrations, say $V_{n}=V_{\text {avg }}=c V_{a}+(1-c) V_{b}$ (here $c N$ is the number of $A$ sites of the chain), and repeats the same calculation in [12], the predicted final velocity is

$$
v=\left[1+V_{\mathrm{avg}}\left(\frac{4 \alpha}{\pi F}\right)^{2}\right]^{-1 / 2},
$$

i.e., the asymptotic velocity is predicted to be smaller (higher) than that of the homogeneous model when $V_{b}>V_{a}$ $\left(V_{b}<V_{a}\right)$.

The results of our numerical simulations are collected in Fig. 1, where we present the value of $v$ as a function of the applied force $F$ for the different orderings considered, always with $V_{b}=10$. The plotted value of $v$ was obtained by starting the simulation for each $F$ with a kink at rest in the middle of the chain, as given by the exact continuum solution of the SG equation, and letting it evolve while monitoring its velocity until it reached a constant value. In all cases it was verified that the soliton shape remained almost unaltered, which is the necessary condition for the concept of velocity to make sense. The simulation parameters are $\alpha=0.1$, which is a prototypical value and whose only effect is to fix the force scale, and $a=0.1$, a value which yields a discrete chain but still close to the continuum to avoid side effects induced by pinning due to the effective PeierlsNabarro potential [17]. In Fig. 1 one easily sees the different behavior of the different kinds of ordering considered. The periodic chain with alternating $V_{a}$ and $V_{b}$ is very accurately described by our theoretical prediction in Eq. (3) with average velocity $V_{\text {avg }}$ corresponding to a concentration of

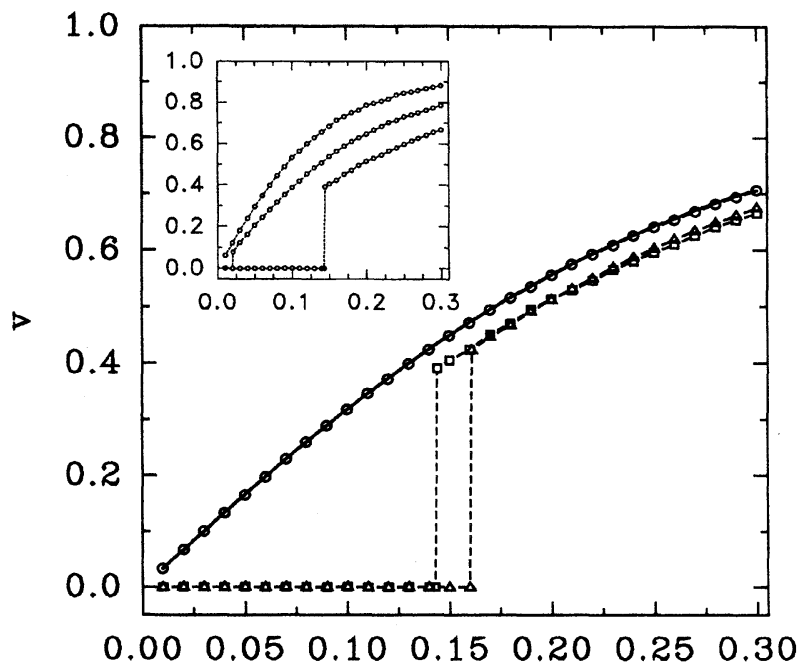

$\mathrm{F}$

FIG. 1. Steady state velocity versus applied force for chains with different orderings and $V_{b}=10$. Points correspond to numerical results, solid lines to the theoretical prediction in Eq. (3) with $V_{\text {avg }}$ corresponds to $c=0.5$. (O), periodic chain with $N=4182$; $(\square)$, Fibonacci chain with $N=F_{18}=4181 ;(\triangle)$, Thue-Morse chain with $N=2^{12}=4096$. Dashed lines joining symbols are a guide to the eye. The threshold for random chains is out of the range of the plot and its value is about $F=0.5$. Inset: same but for Fibonacci chains with $V_{a}=2, V_{a}=5$, and $V_{a}=10$, from top to bottom.

$c=0.5$, whereas it is seen that neither Fibonacci (with $c=\tau$ ) nor Thue-Morse chains obey that equation. The first discrepancy arises as the existence of a threshold force, $F_{c}$, below which solitons are pinned and do not move. A comparison of such $F_{c}$ for the aperiodic chains with two random chains (not shown in the figure) with the same concentration of $V_{a}(\tau=0.618 \ldots$ for the Fibonacci case and 0.5 for the Thue-Morse case) leads to the conclusion that the threshold is about three times higher for the fully disordered chain than for the corresponding aperiodic one. Above threshold, the approximation (3) fails also to accurately predict the value of $v$, overestimating it appreciably for those nonperiodic cases. However, the overall behavior of the random and the aperiodic chains is different, in the sense that in the random case, above threshold the $v-F$ curve is nonmonotonous and the final velocity depends strongly on the particular realization of disorder. As for the dependence of the results on the value of $V_{b}$, it can be seen in the inset of Fig. 1 that, while keeping $V_{b}>V_{a}$, the higher $V_{b}$ the larger $F_{c}$ for Fibonacci lattices. On the other hand, when $V_{b}=0.1$, i.e., much smaller than $V_{a}$, we have found that, contrary to the naive expectations, there is still a threshold force for the Fibonacci case, about $F_{c}=10^{-3}$, which we have verified is not related to Peierls-Nabarro pinning because the periodic chain shows no pinning for such a value of the force. This is a striking result that should be compared to the random chain one, where it is found that $F_{c} \simeq 0.04$

To gain further insight into the pinning phenomenon, we have compared the results for the full aperiodic chains with those of periodic chains with a unit cell formed by a shorter 


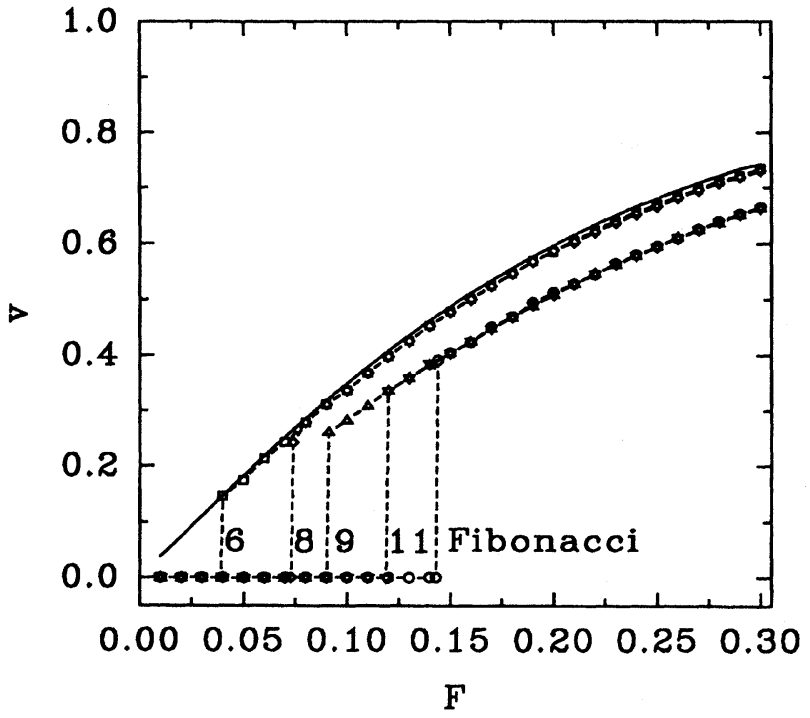

FIG. 2. Steady state velocity versus applied force for chains with different orderings. Points correspond to numerical results, solid lines to the theoretical prediction in Eq. (3) with $V_{\text {avg }}$ corresponding to $c=\tau$. ( $\square)$, sixth approximation (13 sites); ( $\diamond)$, eighth approximation (34 sites); $(\triangle)$, ninth approximation (55 sites); $(\nabla), 11$ th approximation (144 sites); $(\bigcirc)$, full Fibonacci chain. All lengths are close to 4180 sites. A soliton at rest spans about 50 sites for comparison. Dashed lines joining symbols are a guide to the eye. Labels indicate the order of the approximation to the Fibonacci chain as well as the full aperiodic sequence.

approximation to the corresponding aperiodic sequence. Thus, for the Fibonacci chain, we have studied periodic chains with unit cells $V_{a} V_{b} V_{a}$ (the third Fibonacci approximation), $V_{a} V_{b} V_{a} V_{a} V_{b}$ (the fourth approximation), and so forth up to the 11th approximation (144 sites in the unit cell).
The results are shown in Fig. 2. The most remarkable conclusion that can be drawn from this plot is that for periodic chains with unit cells smaller than the soliton size (around 50 sites when at rest), the behavior above threshold is independent of the unit cell, and moreover, it is very well described by the average velocity introduced in Eq. (3). This is very important, since it implies the existence of influences coming from the long-range order of the full aperiodic chain which do not arise in periodic approximations unless the size of the unit cell is much larger than the soliton width, i.e., unless the soliton is unable to distinguish the unit cell from the whole chain. Another remark in order here is that the threshold for the different periodic approximations depends on the initial position of the soliton in the chain, changing up to a factor of 2 for different positions, although keeping below that of the full Fibonacci chain. Results for the Thue-Morse model are basically the same, although in this case shorter lengths are needed for the simulation to get close to that of the full aperiodic chain, and the threshold dependence on the size is nonmonotonous. We tentatively associate this with the fact that in the Thue-Morse sequence $B$ sites may appear in pairs, contrary to the Fibonacci sequence, and therefore it is to be expected that their effect will be stronger on the soliton.

The existence of a threshold force is clearly the main failure of the collective coordinate theory for both the aperiodic and the random chains and, therefore, we undertook the task of finding a better analytical description. To this end, we followed the same approach of the work by Salerno and Kivshar [7], where they introduced an effective potential to account for their results on DNA promoter dynamics. The basic idea is similar to that of the collective coordinate technique, but they improve it by including the spatial ordering of the chain. We skip the details here, as the interested reader may find them in Refs. [6,7], and quote only the final result: The effective potential seen by a soliton, initially at rest at a lattice site $n_{0}$, is given by

$$
W\left(n, n_{0}\right)=\frac{\sum_{m}\left(V_{\mathrm{avg}}+V_{m}\right)\left[\operatorname{sech}^{2}\left(z_{m}\right)-\operatorname{sech}^{2}\left(z_{m}^{(0)}\right)\right]-2 F\left[\tan ^{-1}\left(e^{z_{m}}\right)-\tan ^{-1}\left(e^{z_{m}^{(0)}}\right)\right]}{2 \sum_{m} \operatorname{sech}^{2}\left(z_{m}\right)},
$$

with $z_{m} \equiv a V_{\mathrm{avg}}^{1 / 2}(m-s n)$ and $z_{m}^{(0)} \equiv a V_{\mathrm{avg}}^{1 / 2}\left(m-s n_{0}\right)$, and the sums run over the whole lattice. Finally, to include soliton width effects, the potential in Eq. (4) is averaged in the interval of the lattice spanned by the soliton. We have to stress that this approach only applies to the early stages of the problem, when the kink is at rest or beginning to move at a very slow speed. This is so because in deriving Eq. (4) the dissipative term is not included. Therefore, this approach should be useful in predicting the threshold force although it certainly does not apply to the dynamics above threshold. As can be seen from Fig. 3, the agreement is very good for the Fibonacci chain, and the same can be said about the ThueMorse chain and the random case (not shown; in addition, the random case depends strongly on the realization consid- ered, which was to be expected in view of the simulations). For comparison, notice that the potential minimum is always absent in periodic lattices (see the inset of Fig. 3), in agreement with our numerical simulations where pinning is not observed in those lattices. In computing the average value of $W\left(n, n_{0}\right)$ we have used a soliton width of 40 sites, which is of course quite arbitrary. We have checked that variations of \pm 10 sites are not crucial for our results, which remain semiquantitatively correct. Indeed, due to this freedom in the election of the soliton width, we have not pursued a better agreement, because it would be difficult to justify the choice of that value aside from the fact that it fitted the numerical simulations. 


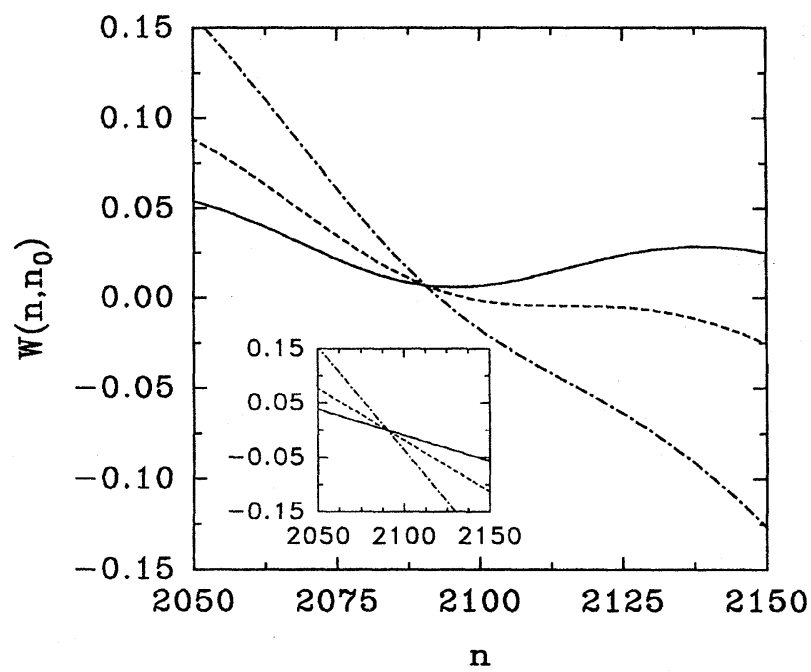

FIG. 3. Effective potentials for a kink soliton initially at rest in the center of a Fibonacci chain for forces $F=0.05$ (solid line), $F=0.1$ (dashed line), and $F=0.2$ (dot-dashed line). Inset: Same, but for the periodic chain.

In conclusion, we have studied soliton propagation along binary lattices with different orderings. We have found that whereas solitons can propagate for any force on periodic systems, nonperiodic ones exhibit a threshold value, i.e., forces larger than a value $F_{c}$ are needed in order to start propagation. We have been able to analytically explain that, as well as to characterize the differences that, in turn, separate the phenomenology of random and aperiodic chains. We have also found that long-range order effects arise when solitons are propagating along the chain, which show up in a decreasing of the steady state velocity with respect to the theoretical expectations for the periodic chains. Our findings are of relevance in a large number of contexts (see, e.g., the book by Davydov [10]). In particular, these results can be useful in the context of Josephson devices, as they can be the basis for the design of new devices with specific properties (the value $F_{c}$ corresponds to a critical current for the device to start conducing). Most importantly, Josephson systems built much in the same way as those in [5] should behave as we have reported, thus providing another check of our results as well as a source of potential new applications. On the other hand, our findings can also be relevant to DNA promoter dynamics (or other soliton phenomena in molecular systems). Indeed, our results show that the long-range correlation present in DNA [11] due to the information it encodes makes soliton propagation easier than if it were purely random, in fact allowing their traveling along the chain at lower velocities. This would not be possible if the structure of the molecule would be random in view of our results.

It is clear that these results just opened the door to the problem of soliton propagation in aperiodic systems. As we have mentioned along the paper, there are a number of unsolved questions, like an analytical explanation of the soliton velocity in aperiodic chains, or how periodic approximations converge to the full aperiodic system. Besides that, the study of other aperiodic models would be helpful in order to clarify the generic properties exhibited by this kind of model. Work along these lines is in progress.

We thank E. Maciá for illuminating conversations on aperiodic systems and J. A. Cuesta, F. Falo, L. M. Floría, P. J. Martínez, and P. Quémerais for helpful discussions, and L. García for the use of his computer facilities. Work at Madrid and Leganés is supported by CICyT (Spain) Project No. MAT95-0325.
[1] A. Sánchez and Luis Vázquez, Int. J. Mod. Phys. B 5, 2825 (1991); S. A. Gredeskul and Yu. S. Kivshar, Phys. Rep. 216, 1 (1992).

[2] Disorder and Nonlinearity, edited by A. R. Bishop, D. K. Campbell, and St. Pnevmatikos (Springer, Berlin, 1989); Nonlinearity with Disorder, edited by F. Kh. Abdullaev, A. R. Bishop, and St. Pnevmatikos (Springer, Berlin, 1992); Fluctuations and Order: The New Synthesis, edited by M. M. Millonas (Springer, Berlin, in press); Fluctuation Phenomena: Disorder and Nonlinearity, edited by A. R. Bishop, S. Jiménez, and L. Vázquez (World Scientific, Singapore, 1995).

[3] A. Barone and G. Paternó, Physics and Applications of the Josephson Effect (Wiley, New York, 1982).

[4] See, e.g., F. Falo, A. R. Bishop, P. S. Lomdahl, and B. Horovitz, Phys. Rev. B 43, 8081 (1991), and references therein.

[5] A. V. Ustinov, M. Cirillo, B. H. Larsen, V. A. Oboznov, P. Carelli, and G. Rotoli, Phys. Rev. B 51, 3081 (1995), and references therein.

[6] M. Salerno, Phys. Rev. A 44, 5292 (1991); M. Salerno, Phys. Lett. A 167, 49 (1992), and references therein.

[7] M. Salerno and Yu. S. Kivshar, Phys. Lett. A 193, 263 (1994).
[8] L. Matsson, Phys. Rev. E 48, 2217 (1993).

[9] A. E. García, Phys. Rev. Lett. 68, 2696 (1992).

[10] S. Roth and H. Bleier, Adv. Phys. 36, 385 (1987); A. S. Davydov, Solitons in Molecular Systems, 2nd ed. (Reidel, Dordrecht, 1991); A. Graja, Low-Dimensional Organic Conductors (World Scientific, Singapore, 1992).

[11] W. Li, T. G. Marr, and K. Kaneko, Physica D 75, 392 (1994), and references therein.

[12] D. W. McLaughlin and A. C. Scott, Phys. Rev. A 18, 1652 (1978).

[13] Yu. S. Kivshar and B. A. Malomed, Rev. Mod. Phys. 61, 763 (1989).

[14] E. Maciá, F. Domínguez-Adame, and A. Sánchez, Phys. Rev. E 50, R679 (1994).

[15] W. H. Press, S. A. Teukolsky, W. T. Vetterling, and B. P. Flannery, Numerical Recipes in $C$, 2nd ed. (Cambridge University Press, New York, 1992).

[16] A. Sánchez, A. R. Bishop, and F. Domínguez-Adame, Phys Rev. E 49, 4603 (1994).

[17] M. Peyrard and M. D. Kruskal, Physica D 13, 88 (1984). 